\title{
Prevalence and analysis of Pseudomonas aeruginosa in chinchillas
}

\author{
Yasuko Hirakawa ${ }^{1 \dagger}$, Hiraku Sasaki ${ }^{*}$, Eiichi Kawamoto ${ }^{2}$, Hiroki Ishikawa $^{3}$, Tetsuya Matsumoto ${ }^{3}$, Naoki Aoyama ${ }^{1}$, \\ Koh Kawasumi ${ }^{1}$, Hiromi Amao ${ }^{1 *}$
}

\begin{abstract}
Background: Chinchillas (Chinchilla laniger) are popular as pets and are often used as laboratory animals for various studies. Pseudomonas aeruginosa is a major infectious agent that causes otitis media, pneumonia, septicaemia enteritis, and sudden death in chinchillas. This bacterium is also a leading cause of nosocomial infections in humans. To prevent propagation of $P$. aeruginosa infection among humans and animals, detailed characteristics of the isolates, including antibiotic susceptibility and genetic features, are needed. In this study, we surveyed $P$. aeruginosa distribution in chinchillas bred as pets or laboratory animals. We also characterized the isolates from these chinchillas by testing for antibiotic susceptibility and by gene analysis.

Results: P. aeruginosa was isolated from $41.8 \%$ of the 67 chinchillas included in the study. Slide agglutination and pulsed-field gel electrophoresis discriminated 5 serotypes and 7 unique patterns, respectively. For the antibiotic susceptibility test, $40.9 \%$ of isolates were susceptible to gentamicin, $77.3 \%$ to ciprofloxacin, $77.3 \%$ to imipenem, and $72.7 \%$ to ceftazidime. DNA analyses confirmed that none of the isolates contained the gene encoding extendedspectrum $\beta$-lactamases; however, 2 of the total 23 isolates were found to have a gene similar to the pill gene that has been identified in the pathogenicity island of a clinical isolate of $P$. aeruginosa.

Conclusions: $P$. aeruginosa is widely spread in chinchillas, including strains with reduced susceptibility to the antibiotics and highly virulent strains. The periodic monitoring should be performed to help prevent the propagation of this pathogen and reduce the risk of infection from chinchillas to humans.
\end{abstract}

\section{Background}

Chinchillas (Chinchilla laniger) are in the Rodentia order, Hystricognathi suborder and the Chinchillidae family. Since chinchillas are small and easy to breed, they are popular as pets. Chinchillas have a large tympanum and cochlear duct similar to the human organ; therefore, they are also often used as laboratory animals for otolaryngological studies [1-4].

Pseudomonas aeruginosa is a Gram-negative rodshaped bacterium and an opportunistic pathogen that causes various serious diseases in humans and animals. Recently, this pathogen has acquired resistance to many therapeutic antibiotics and, in particular, emergence of

\footnotetext{
* Correspondence: h-sasaki@tokyo-med.ac.jp; amao@nvlu.ac.jp

+ Contributed equally

'Laboratory of Experimental Animal Science, Nippon Veterinary and Life Science University, 1-7-1 Musashino-shi, Tokyo, Japan

${ }^{2}$ Animal Research Center, Tokyo Medical University, 6-1-1 Shinjuku-ku, Tokyo, Japan

Full list of author information is available at the end of the article
}

$P$ aeruginosa isolates with extended-spectrum $\beta$-lactamases (ESBLs) has become a serious problem in laboratories and hospitals [5-8]. Thus, it is important not only to investigate the occurrence of $P$. aeruginosa infection but also to assess the existence of antibiotic-resistant $P$. aeruginosa strains to ensure the health of humans and animals. It is known that chinchillas are sensitive to $P$. aeruginosa infection and are therefore frequently used as an animal model of diseases caused by $P$. aeruginosa, including otitis media, otitis interna, pneumonia, septicaemia enteritis, and sudden death [9-12]. In early studies, various clinical treatments against $P$. aeruginosa infection were discovered using chinchillas as an animal model $[11,13]$.

The clinical signs caused by natural infection of $P$. aeruginosa in chinchillas bred as pets or laboratory animals have been described. Doerning et al. [10] reported that the occurrence of $P$. aeruginosa infection in laboratory chinchilla colonies was thought to be
C Biomed Central

(c) 2010 Hirakawa et al; licensee BioMed Central Ltd. This is an Open Access article distributed under the terms of the Creative Commons Attribution License (http://creativecommons.org/licenses/by/2.0), which permits unrestricted use, distribution, and reproduction in any medium, provided the original work is properly cited. 
transmitted by laboratory equipment such as water and water bottles. The clinical signs of $P$. aeruginosa infection have been shown to include otitis media and otitis interna with neurologic manifestations, as well as fatal symptoms in several animals [14]. Although experimental models of $P$. aeruginosa infection in chinchillas have been well described in recent years, there are only few reports on the distribution and abundance of natural infection in chinchillas that are bred as laboratory animals or pets. Furthermore, in these case reports, minimal epidemiological information was supplied.

Typing of $P$. aeruginosa strains and determining the level of susceptibility to antibiotics are epidemiologically useful for measuring the effectiveness of infection control in chinchillas. Minimum inhibitory concentrations (MICs) are helpful indicators to select therapeutic substances and to detect the effects of previously used antibiotics. Zoonoses have long been considered one of the most important threats in public health [15]. To prevent propagation of $P$. aeruginosa infection among humans and animals, detailed characteristics of the isolates, including antibiotic susceptibility and genetic features, may be needed. It is necessary to investigate this epidemiological relationship not only for the health of laboratory animals and pets but also for human health.

In this study, we surveyed the occurrence of $P$. aeruginosa infection among chinchillas bred as laboratory animals or pets. Subsequently, the epidemiological characteristics of $P$. aeruginosa isolates, including serotype and molecular typing, were determined and we tested for the presence of ESBLs in P. aeruginosa isolates by DNA analysis.

\section{Results}

\section{Surveillance of $P$. aeruginosa infection in chinchillas}

A survey was carried out in 67 healthy chinchillas bred both as pets and laboratory animals. Of these animals, 7 of 23 chinchillas bred as pets and 21 of 44 chinchillas bred as laboratory animals were confirmed to be carriers of $P$. aeruginosa. In all, $41.8 \%$ of otherwise-healthy chinchillas were found to be carriers of $P$. aeruginosa. The isolation of $P$. aeruginosa was carried out with swabs from the oral cavity, faeces, and water bottles in the chinchilla cage, with the detection rates of those swabs at approximately $7 \%, 9 \%$, and $20 \%$, respectively. The detection rate of swabs from water bottles showed highly percentage among them. In 2 chinchillas, $P$. aeruginosa was isolated with swabs from the oral cavity, faeces, and water bottles, and in one chinchilla, $P$. aeruginosa was isolated from both oral cavity and faeces.

\section{Typing of $P$. aeruginosa isolates}

Figure 1 shows the dendrogram and results obtained from both serotype and pulsed-field gel electrophoresis
(PFGE) analysis. The serotype of all P. aeruginosa isolates was determined using a slide agglutination test kit. A total of 28 isolates were divided into 4 serotypes (number of isolates): G type (9), B type (11), I type (1), F type (2) and untypeable isolates (5). In the isolates from chinchillas bred as pets, $2 \mathrm{G}$ type and 5 untypeable strains were identified; 11 and 7 B type and $G$ type isolates, respectively, were found in the isolates from chinchillas bred as laboratory animals.

A total of 22 isolates were used to conduct the PFGE analysis because 6 isolates could not be revived after storage. The dendrogram was constructed from the results of the band patterns of the PFGE analysis (Figure 1 ). Seven unique patterns were observed in P. aeruginosa isolates from chinchillas (Additional file 1) and the dendrogram corresponded to these serotypes. The isolates were defined as identical PFGE types if they were $100 \%$ identical. Isolates from the same facility were closely related to each other, indicating that the typing of the isolates by PFGE analysis and serotype contributed to epidemiological data.

\section{Antibiotic susceptibility}

Figures $2 \mathrm{~A}, \mathrm{~B}$ and $2 \mathrm{C}$ show the MICs for aminoglycosides, fluoroquinolones, and selected $\beta$-lactams, respectively. For the aminoglycoside susceptibility test, we selected gentamicin and amikacin (Figures 2). Approximately $60 \%$ of the isolates exhibited an MIC value greater than $8 \mu \mathrm{g} / \mathrm{ml}$ for gentamicin (Figure 2A). According to MIC interpretive standards for $P$. aeruginosa published by the National Committee Clinical and Laboratory Standards (NCCLS) [16], 9 and 13 isolates were susceptible and intermediate to gentamicin, respectively. Only one isolate was intermediate to amikacin according to the NCCL interpretive standards, and none of the isolates showed resistance to these 2 aminoglycosides (Figure 2B). With regard to fluoroquinolones, only one isolate showed an MIC value of $4 \mu \mathrm{g} / \mathrm{ml}$ for ciprofloxacin, while approximately $80 \%$ of the isolates showed an MIC value greater than $4 \mu \mathrm{g} / \mathrm{ml}$ for enrofloxacin (Figure 3A and $3 \mathrm{~B}$ ). According to the NCCL interpretive standards, one isolate was resistant to ciprofloxacin. Prior to sampling, 2 chinchillas bred as laboratory animals were treated with Baytril (enrofloxacin; Bayer, Leverkusen, Germany) for 6 days and Gentacin (gentamicin; Schering-plough animal health, Wim de Körverstraat, Netherlands) for 2 days for otitis media. The MICs of 2 isolates from these chinchillas were 2-4 $\mu$ g/ $\mathrm{ml}$ for enrofloxacin and 4-8 $\mu \mathrm{g} / \mathrm{ml}$ for gentamicin, which may indicate that prior treatment led to the reduced susceptibility to these antibiotics. Except for 2 chinchillas, none of animals was treated with antibiotics.

Further, we tested the $\beta$-lactam antibiotics that have been mainly used for human treatment, including 


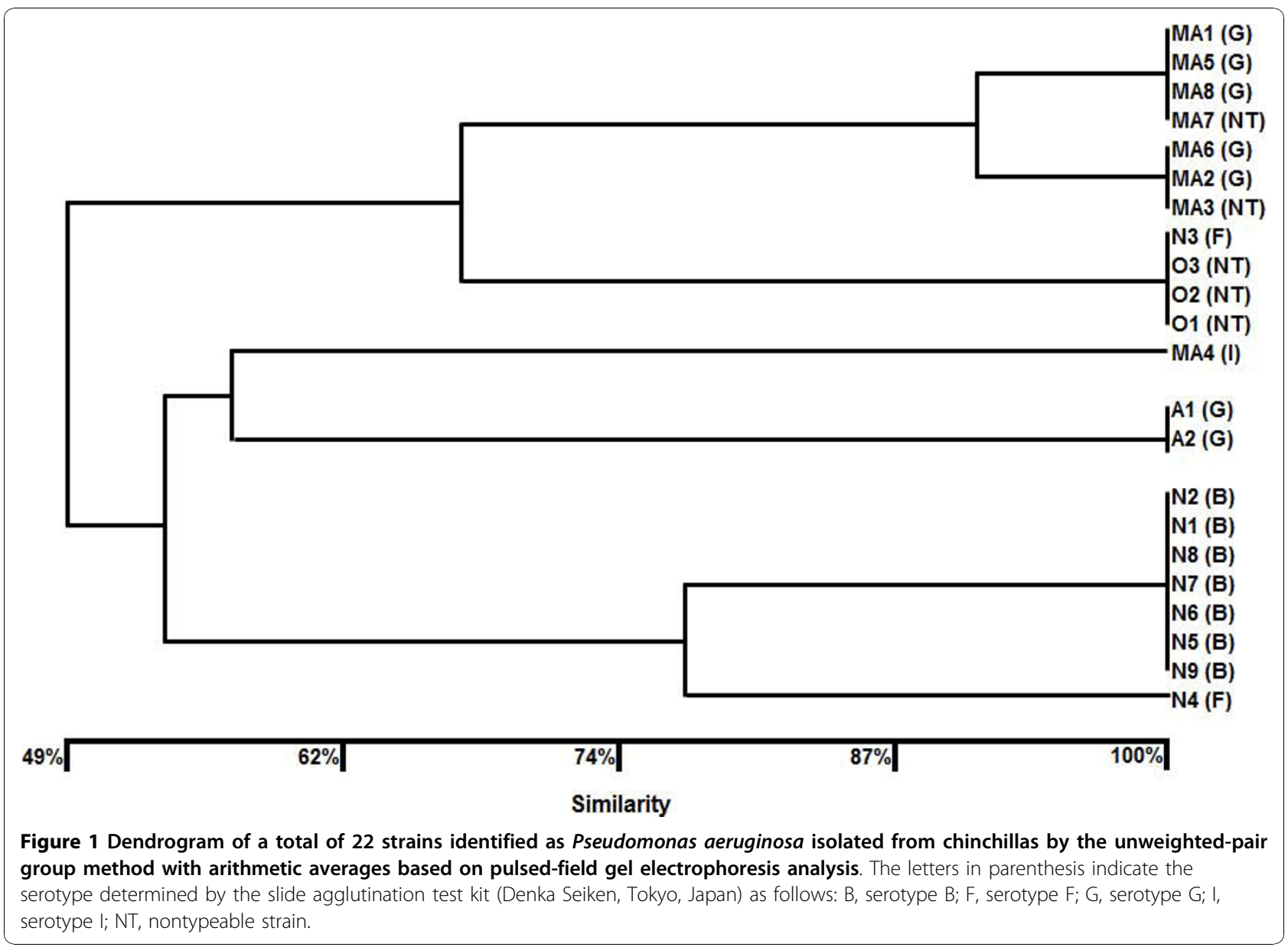

imipenem and third and fourth generation cephalosporins (Figure 4A, B, and 4C). Five isolates that exhibited an MIC of $8 \mu \mathrm{g} / \mathrm{ml}$ were intermediate to imipenem, while 16 isolates showed an MIC value less than $4 \mu \mathrm{g} /$ $\mathrm{ml}$ and were considered susceptible to imipenem according to the NCCL interpretive standards. With regard to cephalosporins, approximately $80 \%$ of the isolates were confirmed to be susceptible to both ceftazidime and cefepime; however, 6 and 1 isolates exhibited an MIC value greater than $16 \mu \mathrm{g} / \mathrm{ml}$ for ceftazidime and cefepime, respectively. For ceftazidime, these 6 isolates were revealed to be intermediate according to the NCCL interpretive standards. These results indicate that the isolates from chinchillas had a reduced susceptibility to the latter antibiotics used for humans, but not to those used for animals.

\section{Polymerase chain reaction (PCR) assay and sequencing}

All isolates underwent PCR amplification to confirm the existence of genes encoding various types of ESBLs in $P$. aeruginosa wild strains $[3,17]$. None of the isolates were found to contain ESBLs by using primer pairs for OXA, TEM, SHV, PER, CTX group 2, CTX group 9 and VEB- type ESBLs. By using primer pairs GES and CTX group1, however, visible bands near the location of expected size were observed in 9 and 2 isolates, respectively (data not shown). To examine sequencing accuracy, TA cloning was performed. By using the GES primer pair, all the inserts that were sequenced were partially identified by blastx search as the ATP-binding cassette (ABC) transporter permease found in various strains of $P$. aeruginosa, while none of the inserts were found to contain the gene encoding the GES-type $\beta$-lactamase. By using the CTX group 1 primer-pair, none of the inserts were found by blastx search to contain the gene encoding the CTX-type $\beta$-lactamase. However, all the inserts from 2 PCR amplicons were highly similar to the gene encoding the putative PilL protein found in P. aeruginosa (accession no. ABR13432), with $82 \%$ coverage and $97 \%$ maximum identity by blastx search. Further, all the inserts were also similar to the gene encoding the type IV B pilus protein in P. aeruginosa (accession no. YP_792920), with $81 \%$ coverage and $95 \%$ maximum identity. Both the PilL and type IV pilus protein were identified in a novel pathogenicity island (PI) in highly virulent clinical isolates, including strain PSE9 and PA14 [18-20]. Although the 

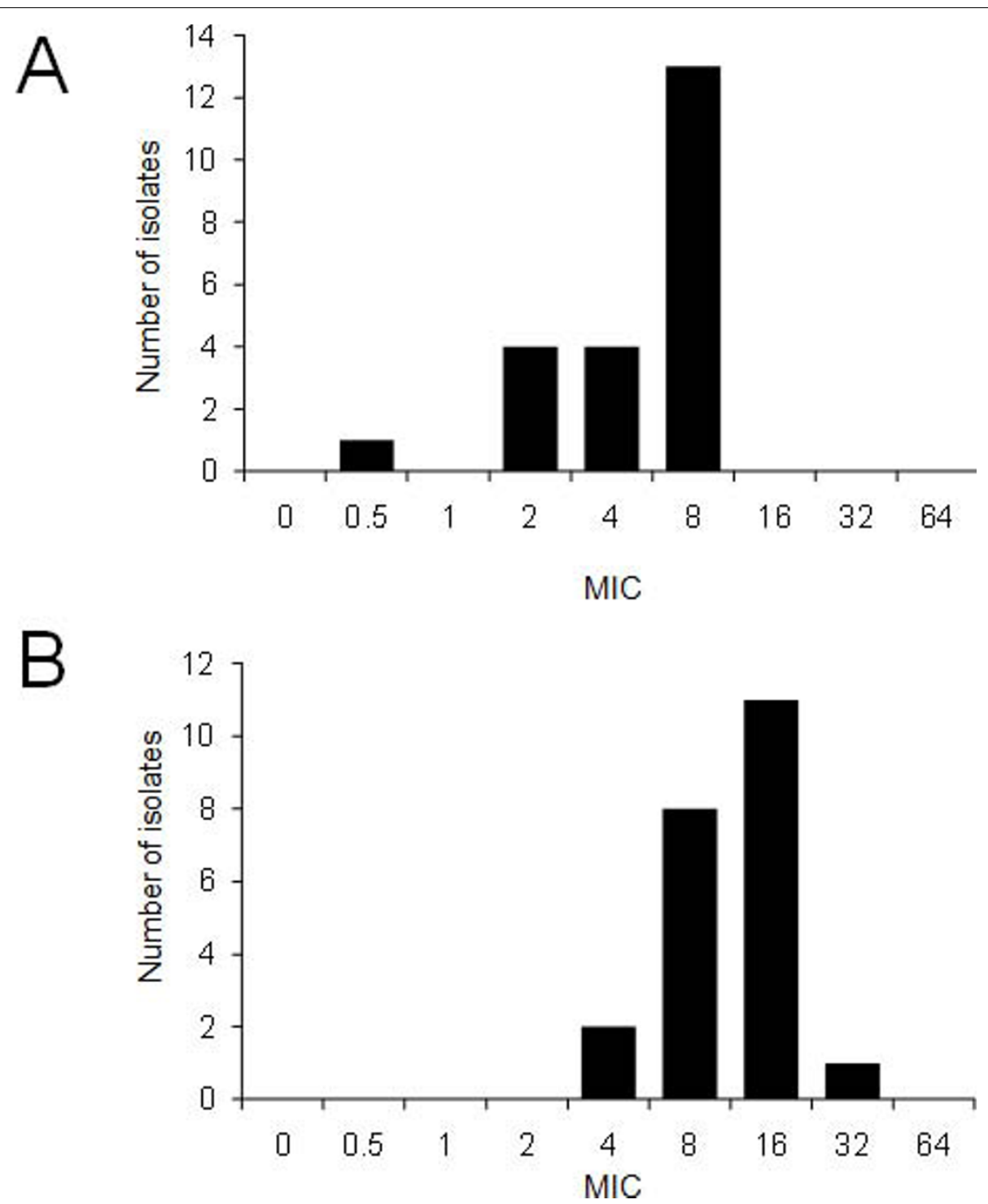

Figure 2 Minimum inhibitory concentrations (MICs) of aminoglycosides in 22 strains of Pseudomonas aeruginosa isolated from chinchillas. (A) Gentamicin (B) Amikacin.

CTX group 1 primer pair may be annealed with a similar sequence but not the targeting gene, the 2 isolates were confirmed to harbour the gene pill. Although we attempted to isolate plasmid from these 2 strains, none of them was confirmed to harbour plasmid. These results also indicate that the 2 isolates containing the gene similar to pilL possibly also possess a PI in their genomes. The two chinchillas that were infected with the $P$. aeruginosa isolates containing the gene similar to pilL were bred as laboratory animals and 2 isolates were found to be susceptible to all tested antibiotics.

Although we also conducted tests to confirm the production of metallo- $\beta$-lactamase (MBL) in all isolates, no isolates were found to produce MBL. Compared with the serotype and the PFGE type, there was no correlation between typing and the susceptibility to antibiotics. Although the genes coding for ESBLs were not detected in chinchilla isolates using PCR assay and sequencing, the 2 isolates containing the gene pilL may be highly virulent strains of $P$. aeruginosa.

\section{Discussion}

In this study, all the chinchillas used for $P$. aeruginosa isolation showed no clinical signs of $P$. aeruginosa infection or other bacterial infections, with the exception of 2 laboratory chinchillas with otitis media. P. aeruginosa was isolated from $30.4 \%$ of pet chinchillas and $47.7 \%$ of laboratory chinchillas. In total, $P$. aeruginosa was isolated 


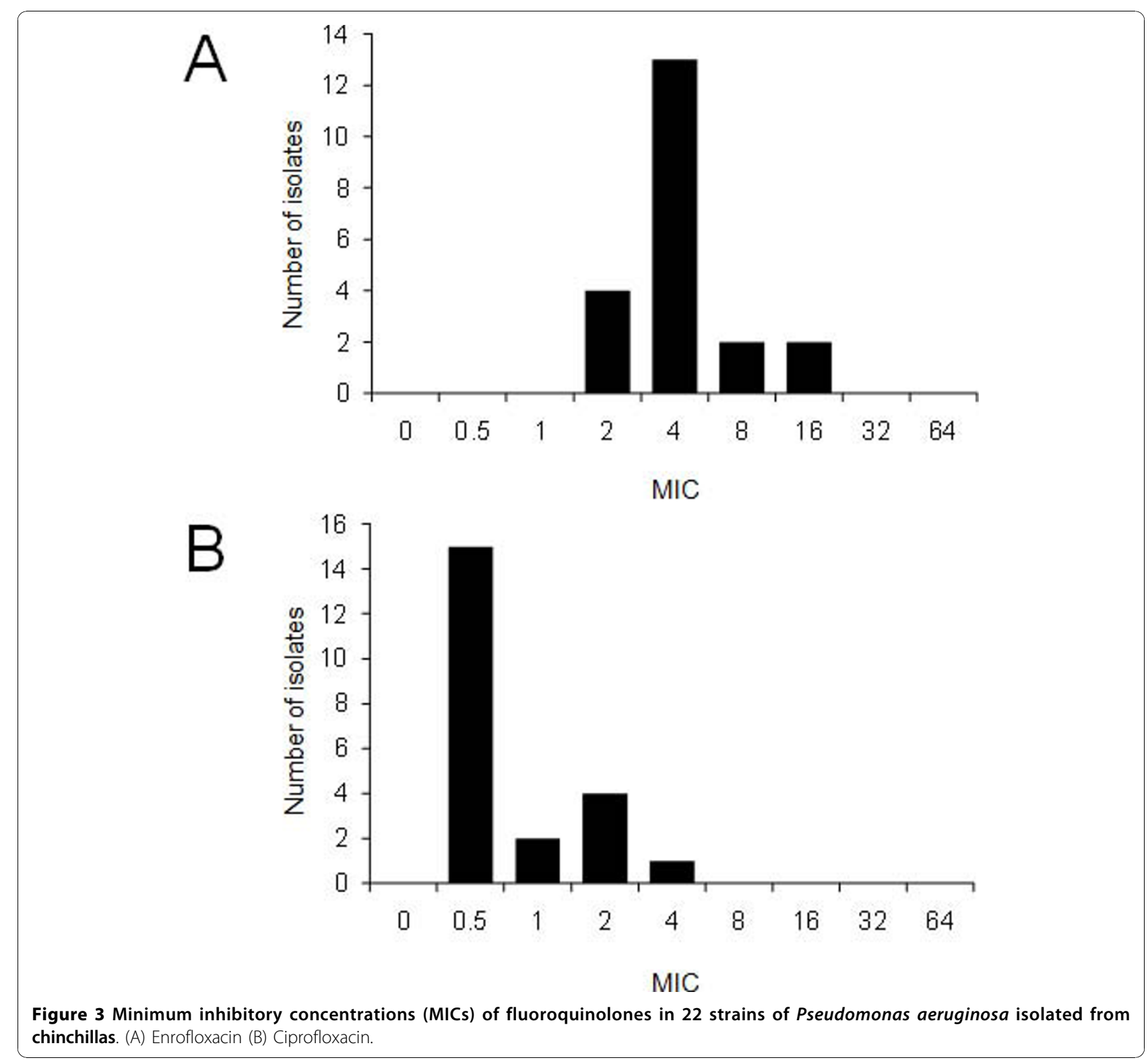

at a rate of $41.8 \%$ in seemingly healthy chinchillas. Although otitis media caused by $P$. aeruginosa and their clinical signs have been reported in many previous studies in chinchillas [9-12], there may be a latent period explaining the lack of such symptoms in the infected animals included in our study. Further, clinical signs of $P$. aeruginosa infection may be developed under immunosuppressive conditions in host animals. Many cases of $P$. aeruginosa infection in chinchillas have been considered to be caused by environmental contaminations such as feed, water and flooring materials. In addition to these contaminations, transient infections may result in silent chronic infections in chinchillas due to coprophagia [21].

Although no evidence was found that the isolates from chinchillas were heterogeneous, serotype and PFGE analyses discriminated the isolates into 5 serotypes and 7 PFGE types, respectively. These results suggest that the isolates diversified despite limited numbers. We used slide agglutination for the determination of serotype as a minor modification of Homma's method [22]. In early epidemiological studies, $P$. aeruginosa isolates from both human and laboratory rodents were reportedly predominantly serotype $\mathrm{G}$, as determined by slide agglutination $[23,24]$. Although serotype $G$ was the second most dominant serotype among the isolates in our study, the isolates belonging to serotype $\mathrm{G}$ were further discriminated into 3 independent PFGE types. Serotype and PFGE type reportedly cross-react with each other using slide agglutination; however, serotype G determined by Homma's method was also divided into 2-4 


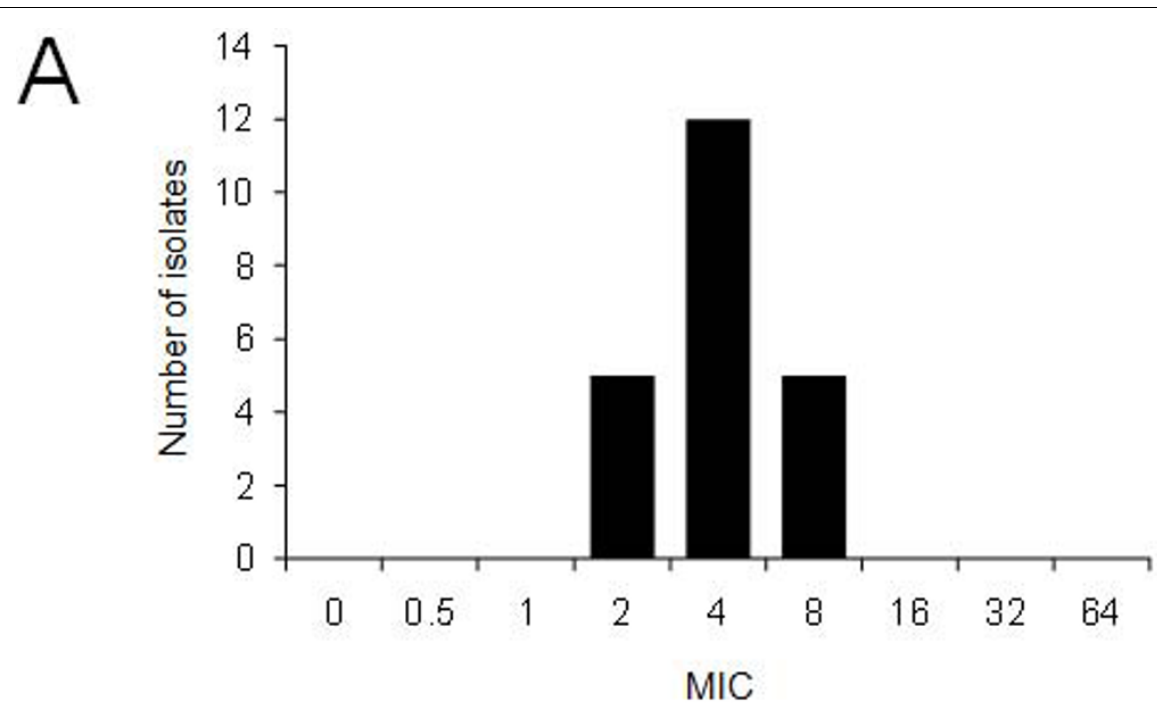

B

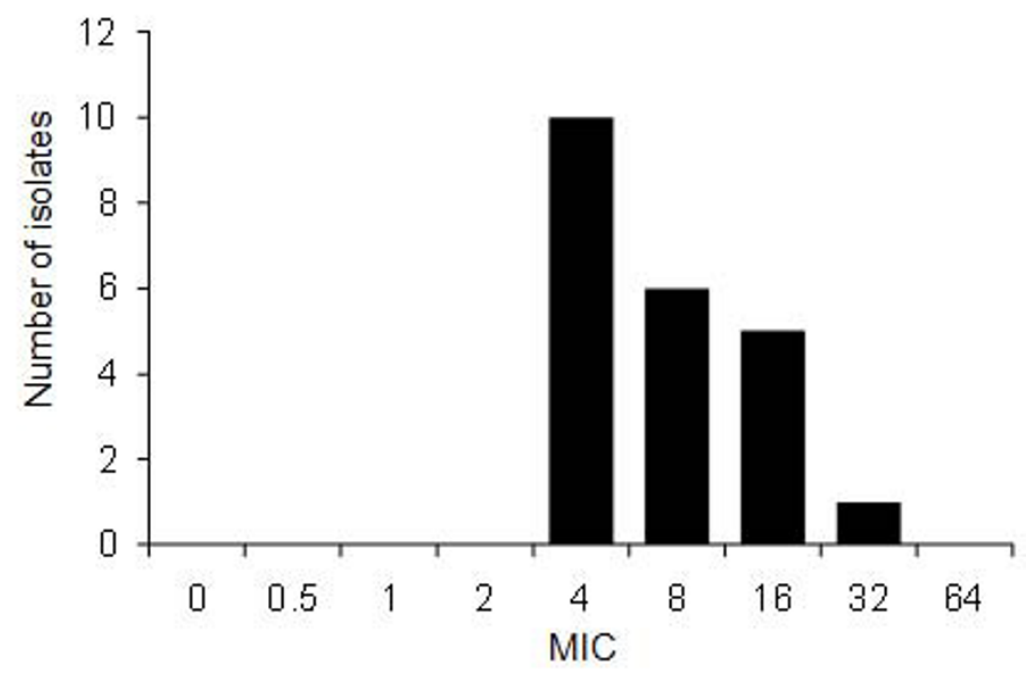

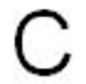

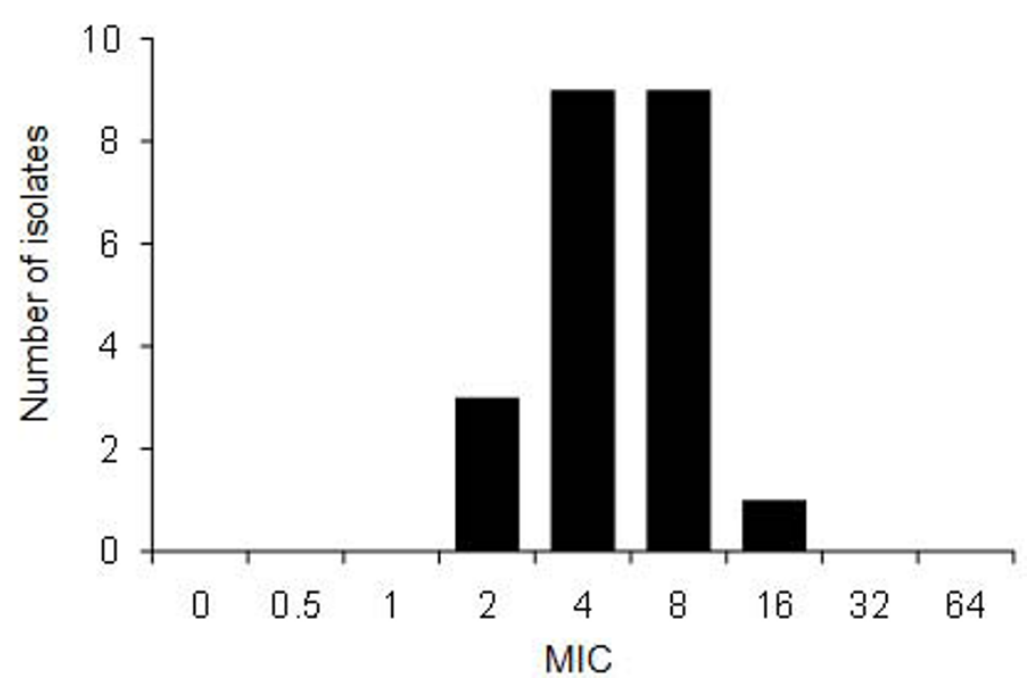

Figure 4 Minimum inhibitory concentrations (MICs) of $\beta$-lactams in 22 strains of Pseudomonas aeruginosa isolated from chinchillas. (A) Imipenem (B) Ceftazidime (C) Cefepime. 
serotypes by other agglutination methods [23]. Thus, there may be no homogeneity in the degree of DNA restriction analysis among these serotypes.

In this study, we determined MICs for 3 different spectrums of antibiotics, including aminoglycosides, fluoroquinolones, and $\beta$-lactams. Prior to the surveillance, all the chinchillas bred as both pets and laboratory animals were confirmed to be receiving no antibiotics, with the exception of 2 laboratory chinchillas. These 2 laboratory chinchillas were treated with enrofloxacin and gentamicin. Although 2 isolates from these animals were considered to be susceptible to enrofloxacin, one of the isolates showed intermediate to gentamicin. These results suggested that the treatment of the antibiotics was led to the reduced susceptibility of the isolate. In laboratory rodents, gentamicin and enrofloxacin have been generally used for the treatment of infectious diseases presumably caused by $P$. aeruginosa or another Gram-negative bacterium [10,25,26], while fluoroquinolones, carbapenems, and cephalosporins, such as ciprofloxacin, imipenem, and cefepime, may not be used for any bacterial infection in laboratory rodents in Japan and are strictly reserved for human use. In our study, no anamneses were found and none of antibiotics were administered in pet chinchillas. Thus, the isolates with reduced susceptibilities to ciprofloxacin, imipenem, and cephalosporins were considered to be transmitted by humans and the environment.

Recently, outbreaks of $P$. aeruginosa infections resistant to various antibiotics have reportedly increased. Of these infections, multidrug-resistant $P$. aeruginosa (MDRP), including EBSL- and MBL-producing P. aeruginosa causing nosocomial infections, has become a serious problem [27-30]. Therefore, the isolates from the chinchillas included in our study were further used for the determination of susceptibility to the antibiotics to which MDRP is resistant. From the antibiotic susceptibility tests, we determined that no isolates exhibited high MIC values for aminoglycosides, fluoroquinolones, or $\beta$-lactams. In particular, none of the isolates showed an MIC value greater than $16 \mu \mathrm{g} / \mathrm{ml}$ for imipenem and none revealed positive results on $\mathrm{MBL}$ tests, indicating that the isolates were not MDRP or MBL-producing P. aeruginosa. However, one isolate exhibited an MIC value greater than 32 $\mu \mathrm{g} / \mathrm{ml}$ for ceftazidime, suggesting that this isolate may have been an ESBL-producing $P$. aeruginosa strain.

In this study, the gene encoding ESBLs could not be identified in $P$. aeruginosa isolates using previously reported primers (Additional file 2). However, the isolates that showed high MICs values for third and fourth generation cephalosporins were considered to have the genetic background for low susceptibility to these antibiotics. These genetic elements should be clarified in future studies. Although the gene encoding ESBLs could not be detected in the isolates using the primer pair CTX group 1, TA cloning identified one of the genes involved in pillin biogenesis. The partially identified gene was similar to the pilL gene that has been reported to be located in the PI of a virulent strain of P. aeruginosa [20]. The PI we observed was also homologous to a previously identified $P$. aeruginosa PI (PAPI-1) that was only found in strain PA14, which is highly virulent for animals and plants $[18,19]$. The genes encoding PilL and type IV $\mathrm{B}$ pilus protein were not identified in less virulent strains of $P$. aeruginosa, such as strain PAO1, but only in highly virulent clinical isolates, including strain PSE9 and PA14. Both proteins were previously found to be related to type IV pillin in the newly discovered PI $[19,20]$. Type IV pillin has been shown to be one of the most important factors for adherence to and invasion of host cells during infection [31]. For P. aeruginosa, the existence of the PI, such as PAPI-1, is considered to be closely related to virulence and pathogenicity for host animals. Thus, monitoring $P$. aeruginosa using genetic information, including the existence of a PI and antibiotic resistance, may help prevent the propagation of the pathogen.

\section{Conclusions}

In this study, $P$. aeruginosa was isolated at high rates from pet and laboratory chinchillas. Typing analysis showed that the isolates were diversified, and the antibiotic susceptibility test showed low susceptibility levels in several isolates. Although we could not identify the gene encoding ESBLs, the isolates were found to contain the gene pilL that was previously identified only in highly virulent strains of $P$. aeruginosa. Thus, it is necessary to take into account the risk of infection from pets or laboratory chinchillas to humans. Because of rapid disease progression and high mortality rates often seen in chinchillas [10], maintaining a clean environment and monitoring should be performed to help prevent $P$. aeruginosa infection.

\section{Methods}

\section{Isolation and identification of $P$. aeruginosa from chinchillas}

The isolation of $P$. aeruginosa was carried in a total of 67 chinchillas, including 23 pets and 44 from 2 breeding facilities for use as laboratory animals. Chinchillas that were used for the isolation of $P$. aeruginosa were all healthy and had no clinical signs, but 1 chinchilla with otitis media was excluded. Further, 2 chinchillas bred as laboratory animals were treated with Baytril for 6 days and Gentacin for 2 days due to otitis media. These 2 laboratory chinchillas recovered completely from otitis media prior to sampling. Oral cavity, faeces, and individual water bottle swabs were collected from each chinchilla and diluted with sterilized saline. The samples 
were spread onto nalidixic acid cetrimide agar (Nissui, Tokyo, Japan) and incubated at $37^{\circ} \mathrm{C}$ for $48 \mathrm{~h}$. Isolates were identified on the basis of colony pigment, Gram staining, and catalase activity. Subsequently, the isolates were identified as $P$. aeruginosa using Quick ID GN"Nissui" (Nissui). The serotype of $P$. aeruginosa isolates was determined with a slide agglutination test kit (Denka Seiken, Tokyo, Japan). Isolates that did not react to any antisera were determined to belong to a nontypeable strain. The glycerol stock of isolates was kept at $-80^{\circ} \mathrm{C}$ until analysis. After this time, 6 isolates could not be revived, and therefore, a total of 22 isolates were used for the analyses. To ensure the accuracy of the identification procedures, 2 reference strains of $P$. aeruginosa ATCC 27853 (American Type Culture Collection, Manassas, VA, USA) and IID 1130 (The Institute of Medical Science, The University of Tokyo, Tokyo, Japan) were used in comparative analyses. Chinchillas were treated in accordance with the provisions for animal welfare of The Nippon Veterinary and Life Science University (formerly The Nippon Veterinary and Animal Science University), which follows the Guidelines for Animal Experimentation issued by the Japanese Association for Laboratory Animal Science.

\section{Pulsed-field gel electrophoresis analysis}

PFGE was carried out as described previously [32,33]. Briefly, all isolates, which were grown in nutrient broth overnight at $37^{\circ} \mathrm{C}$, were centrifuged at $10,000 \mathrm{~g}$ for $2 \mathrm{~min}$. The pellet was resuspended and mixed with $1 \%$ agarose. Lysis was performed at $37^{\circ} \mathrm{C}$ for $1 \mathrm{~h}$, followed by proteinase $\mathrm{K}$ digestion at $50^{\circ} \mathrm{C}$ for $16 \mathrm{~h}$. After 4 washing steps, all plugs were digested with SpeI for $16 \mathrm{~h}$ at $25^{\circ} \mathrm{C}$. After SpeI digestion, the fragments were separated by electrophoresis in $1 \%$ agarose gels with a CHEF-DR apparatus (Bio-Rad, CA, USA) for $18 \mathrm{~h}$, with the switch times ranging from $5.3 \mathrm{~s}$ to $34.9 \mathrm{~s}$ and a field strength of $6 \mathrm{~V} / \mathrm{cm}^{2}$. Lambda ladders were applied as molecular size makers (Bio-Rad). The gels were stained for $20 \mathrm{~min}$ with ethidium bromide and destained for 20 min thereafter. After being photographed under UV, fragment patterns were compared visually and cluster analysis by the unweighted-pair group method with arithmetic averages was performed using NTSYSpc (Exeter Software, NY, USA), as described previously [34].

\section{Antibiotic susceptibility test}

MICs were defined as the lowest concentration of antibiotics that showed no visible growth after $24 \mathrm{~h}$ of incubation at $35^{\circ} \mathrm{C}$. The MICs of antibiotics for all isolates were measured using a broth microdilution technique described by the Clinical and Laboratory Standards
Institute [16]. Antimicrobials were obtained from the following sources: gentamicin (ICN Biomedicals, Aurora, Ohio), amikacin (Bristol Laboratories, Belleville, Ontario, Canada), imipenem (Merck, NJ, USA), ceftazidime (LKT Laboratories, MN, USA), cefepime (Bristol-Myers Squibb, NY, USA), enrofloxacin (MP Biomedicals, $\mathrm{OH}$, USA), and ciprofloxacin (LKT Laboratories). All antimicrobials were thawed and diluted to the desired concentrations with cation-adjusted Mueller-Hinton II broth (BD, MD, USA) prior to each susceptibility test. Each test was repeated at least 3 times. All isolates were assessed for the production of MBLs using metallo- $\beta$ lactamase SMA "Eiken" (Eiken, Tokyo, Japan). To verify the susceptibility of isolates, the MIC values of aminoglycosides, ciprofloxacin, imipenem, and ceftazidime were compared with the description of the NCCL interpretive standards [16].

\section{DNA manipulation}

We used PCR techniques to detect the gene encoding various types of ESBLs found in $P$. aeruginosa using the methods of Jiang et al. [17] and Weldhagen [8]. In brief, PCR was used to detect the gene encoding $\beta$-lactamases, OXA-10, TEM, SHV, PER-1, CTX group 1, CTX group 2, CTX group 9, VEB-1and GES (Additional file 2). The amplicons were confirmed by agarose gel electrophoresis, and visualized bands were subsequently purified with Suprec PCR (Takara bio, Shiga, Japan). The purified PCR products were ligated with T-vector, pTAC-1 using a TA PCR cloning kit (Biodynamics laboratory, Tokyo, Japan), and the resulting plasmids transformed in Escherichia coli DH5 $\alpha$ were spread on Luria-Bertani medium supplemented with $100 \mu \mathrm{g} / \mathrm{ml}$ ampicillin. The colonies were randomly picked up and screened directly for inserts by performing colony PCR with primers M13-f and M13-r (Additional file 2). The PCR products were purified and cycle sequencing reaction was conducted using the BigDye terminator cycle sequencing kit (Applied Biosystems). The products were then analyzed using an ABI 310 DNA sequencer (Applied Biosystems). The closest matches of the sequences were identified by blastx search. For the several isolates, existence of plasmid was confirmed to isolate plasmid DNA using a Miniprep DNA purification kit (Takara). The results were confirmed on agarose gels under UV.

\section{Nucleic acid accession numbers}

The nucleotide sequences of partial genes encoding ABC transporter permease (G-1) and PilL protein $(\mathrm{C}-1)$ were deposited in GenBank through DNA Data Bank of Japan (DDBJ), and the accession numbers assigned were AB591379 and AB591380, respectively. 


\section{Additional material}

Additional file 1: Pulsed-field gel electrophoresis binding patterns of selected reference strains and wild-type strains of Pseudomonas aeruginosa after Spel digestion of total DNA. Lanes: M, Bacteriophage lambda ladder DNA; 1, MA8; 2, MA6; 3, N3; 4, N2; 5, N4; 6, A1; 7, IID 1130; 8, MA4; 9, ATCC 27853.

Additional file 2: Primers used for polymerase chain reaction and sequencing. Nucleotide sequences of primers used for PCR, TA cloning and sequencing were described. ESBL = extended-spectrum $\beta$-lactamase.

\section{Acknowledgements}

We would like to thank the owners of the chinchillas and the laboratories for providing the specimens.

\section{Author details}

${ }^{1}$ Laboratory of Experimental Animal Science, Nippon Veterinary and Life Science University, 1-7-1 Musashino-shi, Tokyo, Japan. ${ }^{2}$ Animal Research Center, Tokyo Medical University, 6-1-1 Shinjuku-ku, Tokyo, Japan. ${ }^{3}$ Department of Microbiology, Tokyo Medical University, 6-1-1 Shinjuku-ku, Tokyo, Japan.

\section{Authors' contributions}

NA performed the survey of $P$. aeruginosa in chinchillas in 2002-2004. YH developed the study, including PFGE analysis and antibiotic susceptibility test. HS performed the PCR assay and sequencing, and coordinated the study. IH and TM supported antibiotic susceptibility of the isolates. EK, HK and HA supported and supervised the study. All authors read and approved the final manuscript.

Received: 4 June 2010 Accepted: 17 November 2010 Published: 17 November 2010

\section{References}

1. Hamernik RP, Patterson JH, Salvi RJ: The effect of impulse intensity and the number of impulses on hearing and cochlear pathology in the chinchilla. J Acoust Soc Am 1987, 81:1118-1129.

2. Snyder DL, Salvi RJ: A novel chinchilla restraint device. Lab Anim 1994, 23:42-44.

3. McFadden SL, Kasper C, Ostrowski J, Ding DL, Salvi RJ: Effects of inner hair cell loss on inferior colliculus evoked potential thresholds, amplitudes and forward masking functions in chinchilla. Hear Res 1998 120:121-132.

4. Zheng XY, McFadden SL, Ding DL, Henderson D: Cochlear de-efferentation and impulse noise-induced acoustic trauma in the chinchilla. Hear Res 2000, 144:187-195

5. Bradford PA: Extended-spectrum beta-lactamases in the 21st century: characterization, epidemiology, and detection of this important resistance threat. Clin Microbiol Rev 2001, 14:933-951.

6. Mavroidi A, Tzelepi E, Tsakris A, Miriagou V, Sofianou D, Tzouvelekis LS: An integron-associated beta-lactamase (IBC-2) from Pseudomonas aeruginosa is a variant of the extended-spectrum beta-lactamase IBC-1. J Antimicrob Chemother 2001, 48:627-630.

7. Poirel L, Weldhagen GF, Naas T, de Champs C, Dove MG, Nordmann P: GES-2, a class A beta-lactamase from Pseudomonas aeruginosa with increased hydrolysis of imipenem. Antimicrob Agents Chemother 2001, 45:2598-2603.

8. Weldhagen GF: Sequence-selective recognition of extended-spectrum $\beta$ lactamase GES-2 by a competitive, peptide nucleic acid-based multiplex PCR assay. Antimicrob Agents Chemother 2004, 48:3402-3405.

9. Doyle WJ: Animal models of otitis media: other pathogens. Pediatr Infect Dis J 1989, 8:S45-S47.

10. Doerning BJ, Brammer DW, Rush HG: Pseudomonas aeruginosa infection in a Chinchilla lanigera. Lab Anim 1993, 27:131-133.

11. Cotter CS, Avidano MA, Stringer SP, Schultz GS: Inhibition of proteases in Pseudomonas otitis media in chinchillas. Otolaryngol Head Neck Surg 1996, 115:342-351.
12. Ehrlich GD, Veeh R, Wang X, Costerton JW, Hayes JD, Hu FZ, Daigle BJ, Ehrlich MD, Post JC: Mucosal biofilm formation on middle-ear mucosa in the chinchilla model of otitis media. JAMA 2002, 287:1710-1715.

13. Lusis PI, Soltys MA: Immunization of mice and chinchillas against Pseudomonas aeruginosa. Can J Comp Med 1971, 35:60-66.

14. Wideman WL: Pseudomonas aeruginosa otitis media and interna in a chinchilla ranch. Can J Vet Res 2006, 47:799-800.

15. Rosen T, Jablon J: Infectious threats from exotic pets: dermatological implications. Dermatol Clin 2003, 21:229-236.

16. National Committee for Clinical Laboratory Standards: Methods for dilution antimicrobial susceptibility tests for bacteria that grow aerobically. National Committee for Clinical Laboratory Standards, Wayne, Pa; 8 2009, approved standard.

17. Jiang $X$, Zhang Z, Li M, Zhou D, Ruan F, Lu Y: Detection of extendedspectrum beta-lactamases in clinical isolates of Pseudomonas aeruginosa. Antimicrob Agents Chemother 2006, 50:2990-2995.

18. He J, Baldini RL, Déziel E, Saucier M, Zhang Q, Liberati NT, Lee D, Urbach J, Goodman HM, Rahme LG: The broad host range pathogen Pseudomonas aeruginosa strain PA14 carries two pathogenicity islands harboring plant and animal virulence genes. Proc Natl Acad Sci USA 2004, 101:2530-2535.

19. Lee DG, Urbach JM, Wu G, Liberati NT, Feinbaum RL, Miyata S, Diggins LT, He J, Saucier M, Déziel E, Friedman L, Li L, Grills G, Montgomery K, Kucherlapati R, Rahme LG, Ausubel FM: Genomic analysis reveals that Pseudomonas aeruginosa virulence is combinatorial. Genome Biol 2006, 7: R90.

20. Battle SE, Meyer F, Rello J, Kung VL, Hauser AR: Hybrid pathogenicity island PAGI-5 contributes to the highly virulent phenotype of a Pseudomonas aeruginosa isolate in mammals. J Bacteriol 2008, 190:7130-7140.

21. Kruckenberg SM, Cook JE, Feldman BF: Clinical toxicities of pet and caged rodents and rabbits. Vet Clin North Am 1975, 5:675-684.

22. Homma JY: Designation of the thirteen O-group antigens of Pseudomonas aeruginosa; an amendment for the tentative proposal in 1976. Jpn J Exp Med 1982, 52:317-320.

23. Zierdt CH, Williams RL: Serotyping of Pseudomonas aeruginosa isolates from patients with cystic fibrosis of the pancreas. J Clin Microbiol 1975, 1:521-526

24. Nakamura N, Noguchi K, Urano T: Elimination of Pseudomonas aeruginosa from an experimental nude mouse colony. Jikken Dobutsu 1994, 43:191-197, (In Japanese with English abstract).

25. Urano T, Maejima K, Okada O, Takashina S, Syumiya S, Tamura H: Control of Pseudomonas aeruginosa infection in laboratory mice with gentamicin. Jikken Dobutsu 1977, 26:259-262, (In Japanese with English abstract).

26. Sasaki H, Kawamoto E, Kunita S, Yagami K: Comparison of the in vitro susceptibility of rodent isolates of Pseudomonas aeruginosa and Pasteurella pneumotropica to enrofloxacin. J Vet Diagn Invest 2007, 19:557-560.

27. Goossens H: Susceptibility of multi-drug-resistant Pseudomonas aeruginosa in intensive care units: results from the European MYSTIC study group. Clin Microbiol Infect 2003, 9:980-983.

28. Paterson DL, Bonomo RA: Extended-spectrum beta-lactamases: a clinical update. Clin Microbiol Rev 2005, 18:657-686.

29. El Solh AA, Alhajhusain A: Update on the treatment of Pseudomonas aeruginosa pneumonia. J Antimicrob Chemother 2009, 64:229-238.

30. Miriagou V, Cornaglia G, Edelstein M, Galani I, Giske CG, Gniadkowski M, Malamou-Lada E, Martinez-Martinez L, Navarro F, Nordmann P, Peixe L, Pournaras S, Rossolini GM, Tsakris A, Vatopoulos A, Cantón R: Acquired carbapenemases in Gram-negative bacterial pathogens: detection and surveillance issues. Clin Microbiol Infect 2010, 16:112-122.

31. Mattick JS: Type IV pili and twitching motility. Annu Rev Microbiol 2002, 56:289-314.

32. Spencker FB, Haupt S, Claros MC, Walter S, Lietz T, Schille R, Rodloff AC: Epidemiologic characterization of Pseudomonas aeruginosa in patients with cystic fibrosis. Clin Microbiol Infect 2000, 6:600-607.

33. Sasaki H, Kawamoto E, Tanaka Y, Sawada T, Kunita S, Yagami K: Comparative analysis of Pasteurella pneumotropica isolates from laboratory mice and rats. Antonie van Leeuwenhoek 2009, 95:311-317.

34. Sasaki H, Kawamoto E, Okiyama E, Ueshiba H, Amao H, Sawada T: Molecular typing of Pasteurella pneumotropica isolated from rodents by amplified ribosomal DNA restriction analysis and pulsed-field gel electrophoresis. Microbiol Immunol 2006, 50:265-272 


\section{doi:10.1186/1746-6148-6-52}

Cite this article as: Hirakawa et al:: Prevalence and analysis of

Pseudomonas aeruginosa in chinchillas. BMC Veterinary Research 2010

6:52.

Submit your next manuscript to BioMed Central and take full advantage of:

- Convenient online submission

- Thorough peer review

- No space constraints or color figure charges

- Immediate publication on acceptance

- Inclusion in PubMed, CAS, Scopus and Google Scholar

- Research which is freely available for redistribution

Submit your manuscript at 